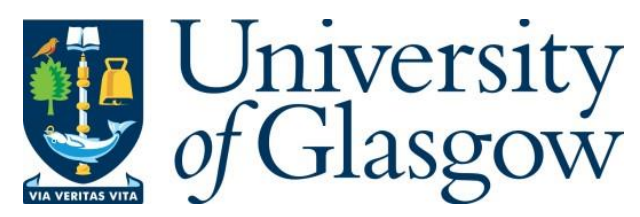

Chen, J., Ghannam, R., Imran, M. and Heidari, H. (2019) Wireless Power Transfer for 3D Printed Unmanned Aerial Vehicle (UAV) Systems. In: IEEE Asia Pacific Conference on Postgraduate Research in Microelectronics and Electronics (PrimeAsia 2018), Chengdu, China, 26-30 Oct 2018, pp. 72-76. ISBN 9781538695913.

There may be differences between this version and the published version. You are advised to consult the publisher's version if you wish to cite from it.

$\underline{\text { http://eprints.gla.ac.uk/168943/ }}$

Deposited on: 14 September 2018

Enlighten - Research publications by members of the University of Glasgow http://eprints.gla.ac.uk 


\title{
Wireless Power Transfer for 3D Printed Unmanned Aerial Vehicle (UAV) Systems
}

\author{
Jiatai Chen, Rami Ghannam, Muhammad Imran and Hadi Heidari \\ School of Engineering, University of Glasgow, G12 8QQ, UK \\ hadi.heidari@glasgow.ac.uk
}

\begin{abstract}
Unmanned aerial vehicles (UAVs) have attracted a lot of attention for various applications such as service delivery, pollution mitigation, farming, and rescue operations over the past few years. However, the short duration of battery and the inconvenience of changing it is always a problem. Basically, small UAVs can only carry very limited payloads otherwise the battery will be drained more frequently. This project presents an automatic and high-efficient wireless power transfer (WPT) to supply a 3D printed UAV. A UAV has been 3D printed with wireless power transfer kit implemented to charge 3S $1500 \mathrm{mAh}$ Li-Po battery with up to $1000 \mathrm{mAh}$ automatically once it is landed, without manual operation. 24V DC is supplied to the transmitting side of WPT with the operating frequency at $180 \mathrm{kHz}$ and once the battery is fully charged, the charging process will also stop automatically.
\end{abstract}

Keywords - Wireless Power Transfer; 3D Printing; Unmanned Aerial Vehicle (UAV).

\section{INTRODUCTION}

Unmanned aerial vehicles (UAVs) plays increasing significance in modern life, with the ability to execute multiple tasks with reliable performance such as surveillance, inspection, filming and delivery. The UAVs can be equipped with many payloads such as a high- resolution camera, infrared camera, sensors, etc., or with objects to deliver. In general, these commercially available systems are powered by a high energy density lithium battery that permits a flight time of about 20-40 min. However, limited battery power obviously restrains UAVs from execute the tasks continuously. Most UAV batteries are not durable due to many factors. Battery technology nowadays has many drawbacks including price, size, weight, charging speed and low energy density. For instance, large batteries are too heavy for a UAV to keep balance or load. UAV with many equipment such as camera will increase the weight, thus increase the power consumption, leading to short battery duration. In this case, users have to charge their UAVs or change the batteries.

To deal with the battery problem, many researchers has made developments in two mainly ways to provide a fully charged battery to UAVs - replacing the battery or recharging it. Wireless Power Transfer [1-7] for UAV Systems Application will reduce the need for users to swap batteries or to connect a power cable, negating the need to return to a single point when battery is drained or any manual operation (Fig. 1). WPT technology permits a very efficient and reliable power

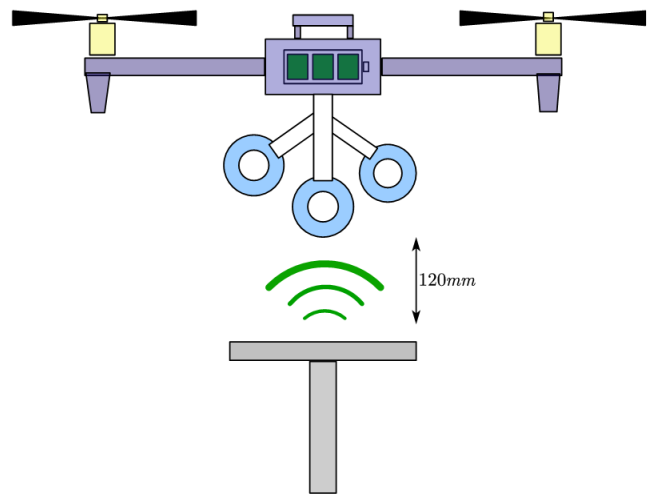

Fig.1. Scheme of wireless power transfer for UAV systems

transmission between the ground base and the UAV. Also, since either the replacing batteries nor exposing the charging contacts to outside environment are required, the UAV can be completely sealed, allowing it to operate in harsh environments. This will enable UAVs to travel further from station and stay in the air longer.

Some researchers also aim at expanding the practicality of WPT technology by increasing transfer power and efficiency [8], by keeping the driving frequency consistent with inherent resonance frequency of LC resonant circuit may improve the behaviour with transmitted power up to $50 \mathrm{~W}$, more than $60 \%$ efficiency at the distance of 1 meter. The state-of-the-art research [9]Error! Reference source not found. is also enhancing the WPT with newly-developed components like wide band-gap transistor diodes or recently discovered inverters is tolerant to load variations and have inherent voltage or current regulation features enable an WPT system to operate effectively.

To cope with battery problem, Wireless Power Transfer Technology is utilized. Here, a novel angle is proposed to improve the battery duration and efficiency by utilizing WPT technology aiming at becoming less sensitive to the misalignment of the coils with 3D printing technology, in addition to reduce the complexity of fabrication and total weight of UAV. The proposed solution consists of a ground transmitting coil module with multiple receiving on-board coils to charge $3 \mathrm{~S}$ Li-Po battery and a self-designed pad with extended support for secondary coils with 3D printing technology. The theories of WPT, the reason why 3D printing is better or why I would like to transfer power wirelessly to UAV and method of design and fabrication will now be described. 


\section{Wireless Power Transfer}

Wireless Power Transfer (WPT) is a term defined as transmitting electromagnetic energy through a physical electromagnetic field. [10]

WPT can be categorized into two types, based on power transmission distance, near-field or far-field, as the former one is used in this project. If the transfer distance is longer than the wavelength of electromagnetic wave, it is categorized into farfield technique. Although far-field WPT have transmission range over kilometres, they have disadvantages of directionality and poor efficiency. Also their operational frequency range are typically very high(GHz range). Naturally, near-field WPT refers to the distance of power transfer within the wavelength of the transmitter antenna. With different types of coupling techniques used, near-field WPT can be categorized into four: (1) inductive coupling, (2) magnetic resonant coupling, (3) capacitive coupling, and (4) magneto dynamic coupling. The first one is utilized in this solution.

Inductive coupling is based on a simple principle: A primary coil is connected to a source, driving the varying magnetic field. The primary transmitter coil will induce a voltage across the secondary receiver coil and transfer the power to the load accordingly. This can be utilized to transfer high power with significant efficiency in a very near distance up to several centimetres [11].

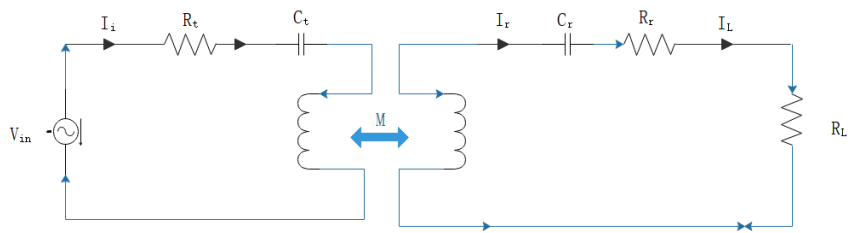

Fig. 2. WPT system equivalent circuit

Typically, two types of resonant coils are used in WPT: LC resonators and self-resonators. LC resonators has an externally added capacitor while Self type resonate with selfinductance of coil and parasitic capacitance between turns, which has the advantage of low loss but it will be pretty difficult to realize this in low frequencies. LC resonators are more controllable. Using Kirchhoff's voltage law, Eq(1) can be obtained.

$$
Z_{t, r}=R_{t, r}+j \omega L_{t, r}+\frac{1}{j \omega C_{t, r}}
$$

where $Z_{t}, Z_{r}$ are self-inpedances of transmitter and receiver resonators respectively.

If we express the mutual inductance(M) between the primary and secondary coils in terms of coupling coefficient $(\mathrm{k})$ and coil inductances,

$$
\mathrm{M}=\mathrm{k} \sqrt{L_{t} L_{r}}
$$

Where $\mathrm{L}_{\mathrm{t}, \mathrm{r}}, \mathrm{C}_{\mathrm{t}, \mathrm{r}, \mathrm{r}}, \mathrm{R}_{\mathrm{t}, \mathrm{r}}$ are inductance capacitance and resistance of the coils respectively.

Output power at the load is:

$$
P_{\text {out }}=\left|I_{L}\right|^{2} R_{L}
$$

And the power transfer efficiency (PTE) is

$$
\begin{gathered}
\text { PTE }=\frac{\left|I_{L}\right|^{2} R_{L}}{\left|I_{L}\right|^{2} R_{t}+\left|I_{L}\right|^{2}\left(R_{L}+R_{r}\right)} \\
I_{i}=\frac{\left(R_{L}+Z_{r}\right) V_{S}}{\left(Z_{t}+R_{S}\right)\left(R_{L}+Z_{r}\right)+(\omega M)^{2}} \\
I_{L}=\frac{(j \omega M) V_{S}}{\left(Z_{t}+R_{S}\right)\left(R_{L}+Z_{r}\right)+(\omega M)^{2}}
\end{gathered}
$$

When in resonance, reactive impedance of the coil turns to zero. Resonance frequencies of the primary and secondary coils becomes.

$$
\begin{gathered}
X_{t, r}=j \omega_{t, r} L_{t, r}+\frac{1}{j \omega_{t, r} C_{t, r}} \\
=0 \\
\omega_{t, r}=\frac{1}{\sqrt{L_{t, r} C_{t, r}}}
\end{gathered}
$$

It can be told from Figure 2 that the maximum Power Transfer Efficiency occurs at the resonance frequency (f0) for a two-coil system. In addition, the Efficiency also increases with the coupling coefficient $\mathrm{k}$ between the primary and secondary coils.

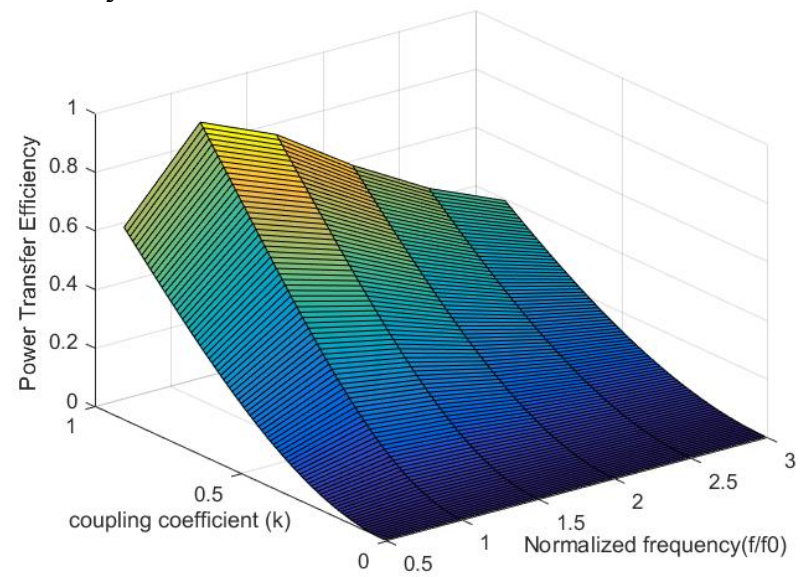

Fig. 3. Power Transfer Efficiency variation with respect to normalized frequency $(\mathrm{f} / \mathrm{f} 0)$ and coupling coefficient $(\mathrm{k})$.

At resonance condition, the frequency is $\omega_{0}=\frac{1}{\sqrt{L_{t} C_{t}}}=\frac{1}{\sqrt{L_{r} C_{r}}}$ and the efficiency can be denoted as

$$
P T E_{\omega_{0}}=\frac{R_{L}\left(\omega_{0} M\right)^{2}}{\left(\left(R_{t}+R_{S}\right)\left(R_{L}+R_{r}\right)+\left(\omega_{0} M\right)^{2}\right)\left(R_{L}+R_{r}\right)}
$$

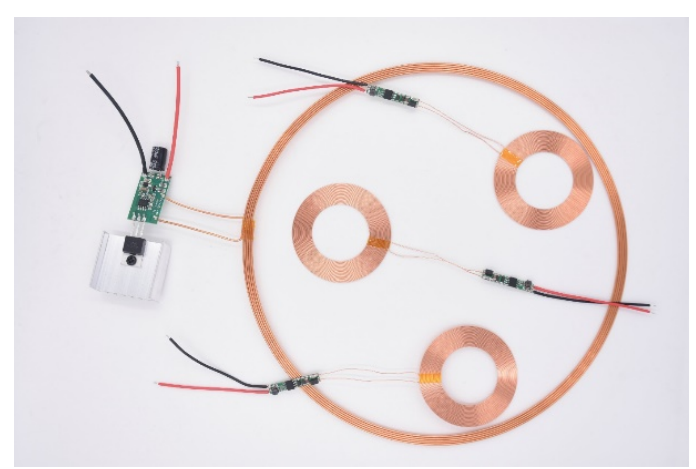

Fig. 4. wireless power transfer module 


\section{DESIGN AND FABRICATION}

Wireless power transfer module XKT-801 (Figure 3) is utilized to compose the WPT system, which requires $24 \mathrm{~V} \mathrm{DC}$ input to the transmitting coil module. Within the chip of primary coil module has a inverter to convert the DC signal to AC signal for inductor to generate electromagnetic field to transmit power. At secondary coil side, AC signal needs to convert back to DC for the battery. The embedded rectifier is used to do this job. After rectifying the AC signal to $\mathrm{DC}$, the $\mathrm{DC}$ voltage at the receiving end is measured as,

Table 1. Distance Vs. receiving side output Voltage

\begin{tabular}{|c|c|}
\hline distance $(\mathrm{mm})$ & Voltage(V) \\
\hline 59.7 & 5.04 \\
\hline 80.9 & 5.05 \\
\hline 82.5 & 5.04 \\
\hline 104.21 & 5.05 \\
\hline 114.9 & 5.04 \\
\hline 129.4 & 4.95 \\
\hline 145.9 & 4.07 \\
\hline 163.4 & 3.26 \\
\hline 185.11 & 2.66 \\
\hline 219.11 & 2.28 \\
\hline
\end{tabular}

distance(mm) VS Voltage(V)

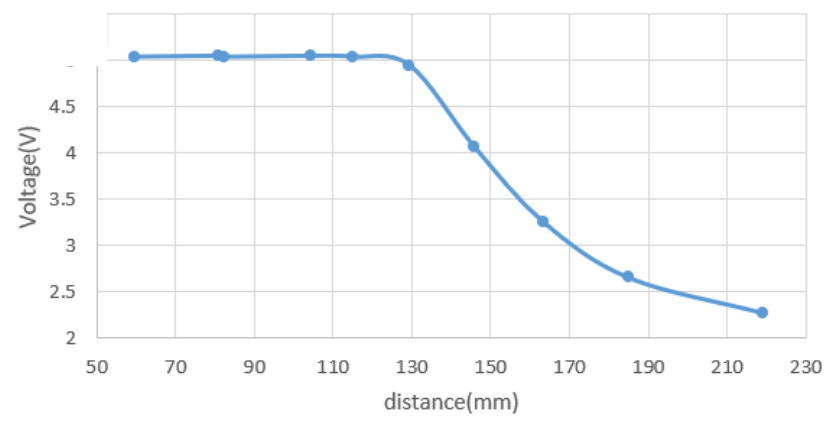

Fig. 5. the output voltage versus distance between transmitting and receiving coil.

The output voltage from the Wireless Power Transfer System cannot charge the battery directly. A balance charger is needed to ensure the several-cells-in-series Li-Po battery can be discharged and charged properly.

The basic principle of balance charging is to detect the difference between the voltage of different cells and charge them separately with either large current for low-voltage cells or small current for high-voltage cells. In this case, TP4056 is selected as the battery charging module.

Because we are using the $3 \mathrm{~s} \mathrm{Li-po} 1500 \mathrm{mAh}$ battery, which is composed of three cells in series. Fig. 6(a) shows the internal connection of the battery. As a result, three TP4056 in series is needed to balance charge this battery. Also, the charging current is changed from the default value of
$1000 \mathrm{mAh}$ to $520 \mathrm{mAh}$ by changing $\mathrm{R} 3$ with a resistor of $2.3 \mathrm{k} \Omega$. This is because the greater normal charging current does not bring better charging result or may even do harm to the Li-Po battery.
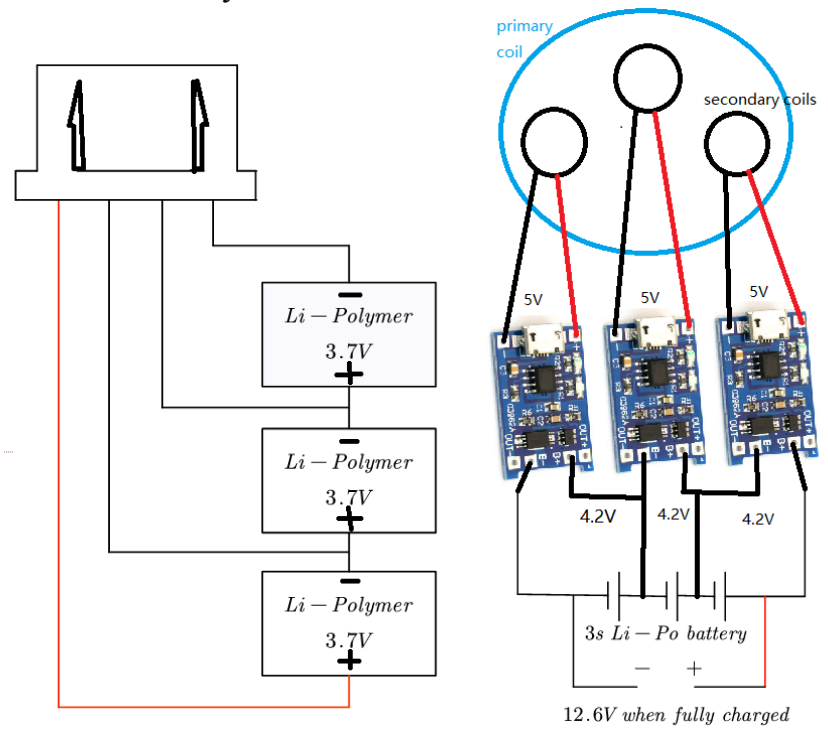

Fig. 6. (left). Internal connection of $3 \mathrm{~S} \mathrm{Li-Po} \mathrm{battery.} \mathrm{(right)}$ Configuration of WPT System with battery charging module.

The required input of the TP4056 module is $5 \mathrm{~V} \mathrm{DC}$, which is another important reason why this module is chosen--- the output from the receiving coil module of Wireless Power Transfer System is exactly $5 \mathrm{~V}$ within the operating distance of $12 \mathrm{~cm}$, according to Table 1. As shown in Fig. 6(b), three receiving coils are utilized to provide $5 \mathrm{~V}$ DC input for three TP4056 separately. This configuration will balance charge three cells of the $3 \mathrm{~S} \mathrm{Li-Po}$ battery. After several experiments, the TP4056 will monitor the voltage of cells and change the charging current accordingly.

In a previous work [12], the on-board coil has been placed on the landing skid. Such a solution permits an enhancement of the system performance due to the increase of the coupling factor between the coils by reducing the vertical air gap between the transmitting and receiving coils. However, it requires high accuracy of alignment. Its main drawback is the poor robustness to the misalignment condition because of the small dimension of the receiving coil. This problem maybe pretty critical as landing precision is relatively hard to control in UAV applications since it depends much on the landing algorithm or environment or landing assistance system.

Additionally, in order to ensure the proper transmission of energy and the stability of electromagnetic field, there must be no metal in the vertical space of the receiving coil, which obviously include most of the components on the UAV, like the battery, power distribution board or flight control.

In the proposed new configuration, the receiving coil is placed on an extended support from the board. Accurate landing is no longer necessary as long as the receiving coil is in the vertical space of the primary coil. This should significantly reduce the complexity of landing algorithm or 
stringent requirement of landing point as well as reduce the effect of metal components on power transmission.

3D printing technology is utilized to build the extended support. 3D Printing allows makers to manufacture personalized products according to an individual's needs and requirements. This increases the feasibility of making customized UAVs. Any specific part of a store-bought UAV can also be tailored to suit personal needs. As a WPT system is required to be mounted onto the UAV, there was a slight modification to the stl file and managed to build an extended support for the receiving coils, as shown in Fig. 7. In this case, there is no need to find an alternative stick, drill a hole on the board and connect them with screws. Obviously using 3D printing technology saves plenty of time in manufacturing.

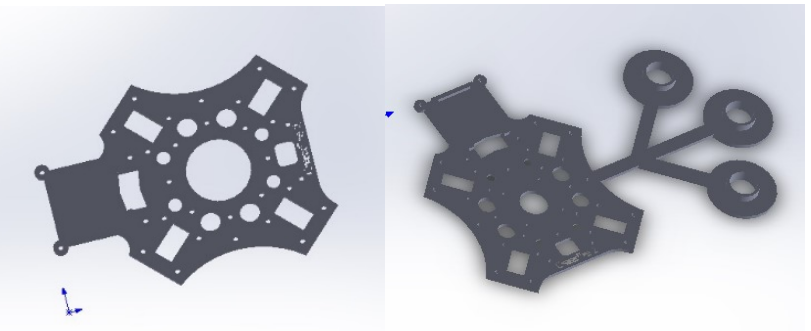

Fig. 7. original file and modified file with extended support for receiving coils.

\section{EXPERIMENTAL RESUltS}

A UAV based on 3D-Printed frame is fabricated and implemented with the WPT system to test the behaviour of wireless power transfer and battery charging. First, the UAV is manually operated, until it flies near the transmitting coil module on the ground, connected to $24 \mathrm{~V}$ DC source. When the UAV approaches the transmitting coil, the LED on battery charging module showing the battery starts to be charged. After landing at the ground for charging, the UAV is then started again. When UAV leaves the operating distance of the WPT system, the LED goes blue and the battery charging is cut-off.

According to experiments, a distance up to $12 \mathrm{~cm}$ is within the efficient operating zone for the wireless power transfer system. When primary coils on the ground and the on-board receiving coils are separated beyond this distance, charging process will stop. When transmitter and receivers are close enough to the effective range again, charging process will start. It can be seen from the Fig. 8 that the Li-Po battery is connected to the battery charging module. The red LED shows the battery is being charged. From the zoom-in part of DC power source, the input power is $2.76 \mathrm{~W}$. If the voltage of cells is lower than $4.2 \mathrm{~V}$, battery charging module TP4056 will charge battery with set current according to Rx (Section 4.2). When the battery voltage is approaching the limit value of battery charging module, the LED will turn blue and charging current will turn to zero, thus cut off the charging.

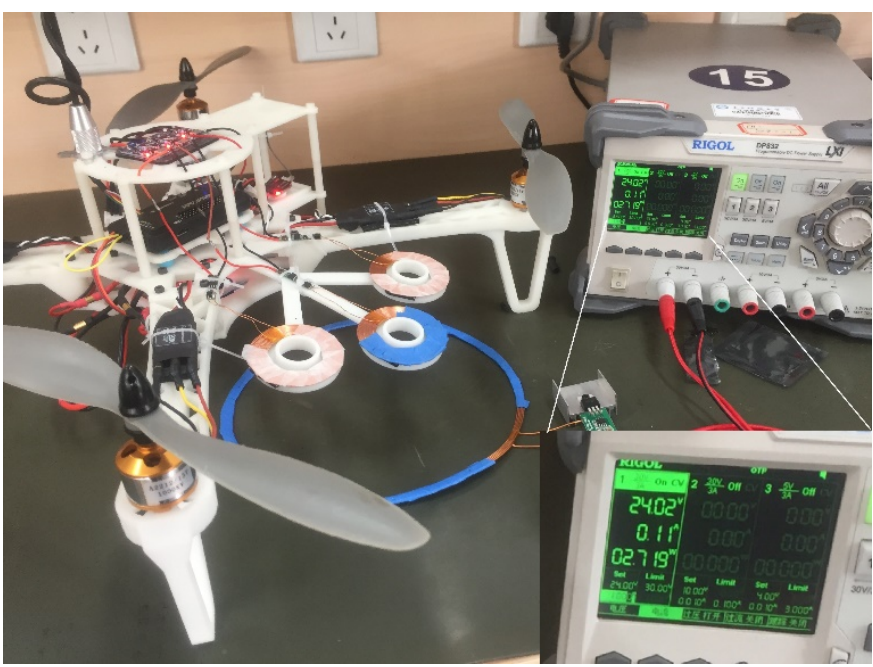

Fig. 8. Experiment result.

\section{Conclusion}

In this paper, a UAV and a Wireless Power Transfer System is fabricated and applied to it to fulfil the purpose of charging the UAV without manual operation or cable connection. The basic theories of WPT and analysis based on numerical methods are also introduced to make decision on components selection, designing, and utilization. 3D printing technology has been utilized to improve the behaviour the UAV while not impairing the strength of material and also reduce the complexity of fabrication, which is meaningful in mass production or individual application.

This proposed solution is also significant in providing a possibility to build an autonomous system [13-17]. It is based on WPT System on UAV application that UAV with routine mission like surveillance, monitoring or couriers can finish their job and return to charging station when battery is drained. After a short time of charging, UAVs can repeat the mission again, while the whole process is manual-operation-free. This may greatly reduce the cost of labour and more workers can put in meaningful jobs instead of connecting UAVs to power cables.

\section{References}

[1] Covic, G.A.; Boys, J.T. Inductive power transfer. Proc. IEEE 2013, 101, 1276-1289.

[2] Shinohara, N. Power without wires. IEEE Microw. Mag. 2011, 11, 64-73.

[3] Campi, T.; Cruciani, S.; Maradei, F.; Feliziani, M. Near Field reduction in a Wireless Power Transfer System using LCC compensation. IEEE Trans. Electromagn. Compat. 2017, 59, 686-694.

[4] Campi, T.; Cruciani, S.; De Santis, V.; Feliziani, M. EMF safety and thermal aspects in a pacemaker equipped with a wireless power transfer system working at low frequency. EEE Trans. Microw. Theory Tech. 2016, 64, 375-382.

[5] Jawad, A.M.; Nordin, R.; Gharghan, S.K.; Jawad, H.M.; Ismail, M. Opportunities and Challenges for Near-Field Wireless Power Transfer: A Review. Energies 2017, 10, 1022. 
[6] Vijayakumaran Nair, V.; Choi, J.R. An Efficiency Enhancement Technique for a Wireless Power Transmission System Based on a Multiple Coil Switching Technique. Energies 2016, 9, 156.

[7] Feliziani, M.; Campi, T.; Cruciani, S.; Maradei, F.; Grasselli, U.; Macellari, M.; Schirone, L. Robust LCC compensation in wireless power transfer with variable coupling factor due to coil misalignment. In Proceedings of the 2015 IEEE 15th International Conference on Environment and Electrical Engineering (EEEIC), Rome, Italy, 10-13 June 2015.

[8] C. Zhu, K. Liu, C. Yu, R. Ma, and H. Cheng, Simulation and experimental analysis on wireless energy transfer based on magnetic resonances, in Vehicle Power and Propulsion Conference, 2008. VPPC '08. IEEE, sept. 2008, pp. $1-4$.

[9] Samer Aldhaher, Paul D. Mitcheson, Juan M. Arteaga, George Kkelis and David C. Yates, Light-Weight Wireless Power Transfer for Mid-Air Charging of Drones, 2017 11th European Conference on Antennas and Propagation (EUCAP)

[10] AM Jawad, R Nordin, SK Gharghan, HM Jawad, Opportunities and Challenges for Near-Field Wireless Power Transfer-A Review, 2017

[11] Shinohara, N. History, Present and Future of WPT, in Wireless Power Transfer Via Radiowaves; John Wiley \& Sons, Inc.: Hoboken, NJ, USA, 2013

[12] T Campi, S Cruciani, M Feliziani,Wireless Power Transfer Technology Applied to an Autonomous Electric UAV with a Small Secondary Coil, Energies2018, 11, 352.

[13] V. Nabaei, et al., "Magnetic Biosensors: Modelling and Simulation," Biosensors and Bioelectronics, vol. 103, pp. 6986, 2018.

[14] H. Fan, et al., "High resolution and linearity enhanced SAR ADC for wearable sensing systems," in IEEE Int. Symp. Circuits Systems (ISCAS), 2017, pp. 1-4.

[15] V. Nabaei, et al., "Magnetic Biosensors: Modelling and Simulation," Biosensors and Bioelectronics, vol. 103, pp. 6986, 2018.
[16] V. Nabaei, et al., "Magnetic Biosensors: Modelling and Simulation," Biosensors Bioelectronics, vol. 103, pp. 69-86, 2018.

[17] K. O. Htet, et al., "Switched Capacitor DC-DC Converter for Miniaturised Wearable Systems," in 2018 IEEE International Symposium on Circuits and Systems (ISCAS), 2018, pp. 1-5.

[18] K.-M. Lei, et al., "A Handheld High-Sensitivity Micro-NMR CMOS Platform With B-Field Stabilization for Multi-Type Biological/Chemical Assays," IEEE J. Solid-State Circuits, vol. 52, pp. 284-297, 2017.

[19] S. Samyak, et al., "On-chip Magnetoresistive Sensors for Detection and Localization of Paramagnetic Particles," in Proc. of IEEE SENSORS Conference, Glasgow, UK, 2017.

[20] X. Liang, et al., "Wearable Capacitive-Based Wrist-Worn Gesture Sensing System," in IEEE New Generation Circuits Systems (NGCAS), 2017, pp. 181-184.

[21] S. Wen, H. Heidari, et al., "A Wearable Fabric-Based RFID Skin Temperature Monitoring Patch," in Proc. of IEEE Sensors Conference, 2016.

[22] S. Samyak, et al., "On-chip Magnetoresistive Sensors for Detection and Localization of Paramagnetic Particles," in Proc. of IEEE SENSORS Conference, Glasgow, UK, 2017.

[23] X. Liang, et al., "Wearable Capacitive-Based Wrist-Worn Gesture Sensing System," in IEEE New Generation Circuits Systems (NGCAS), 2017, pp. 181-184.

[24] S. Samyak, et al., "On-chip Magnetoresistive Sensors for Detection and Localization of Paramagnetic Particles," in Proc. of IEEE SENSORS Conference, Glasgow, UK, 2017.

[25] X. Liang, et al., "Wearable Capacitive-Based Wrist-Worn Gesture Sensing System," in IEEE New Generation Circuits Systems (NGCAS), 2017, pp. 181-184. 